\title{
Unenhanced CT imaging is highly sensitive to exclude pheochromocytoma: a multicenter study
}

\author{
Edward Buitenwerf', Tijmen Korteweg², Anneke Visser', Charlotte M S C Haag², Richard A Feelders³, \\ Henri J L M Timmers ${ }^{4}$, Letizia Canu ${ }^{4,5}$, Harm R Haak ${ }^{6,7,8}$, Peter H L T Bisschop ${ }^{9}$, Elisabeth M W Eekhoff ${ }^{10}$, \\ Eleonora P M Corssmit ${ }^{11}$, Nanda C Krak ${ }^{12}$, Elise Rasenberg ${ }^{13}$, Janneke van den Bergh ${ }^{14}$, Jaap Stoker ${ }^{15}$, \\ Marcel J W Greuter ${ }^{2}$, Robin P F Dullaart ${ }^{1}$, Thera P Links ${ }^{1}$ and Michiel N Kerstens ${ }^{1}$
}

\begin{abstract}
Departments of ${ }^{1}$ Endocrinology, ${ }^{2}$ Radiology, University of Groningen, University Medical Center Groningen, Groningen, The Netherlands, ' ${ }^{3}$ Department of Endocrinology, Erasmus Medical Center, Rotterdam, The Netherlands, "4Section of Endocrinology, Department of Internal Medicine, Radboud University Medical Center, Nijmegen, The Netherlands, ${ }^{5}$ Department of Experimental and Clinical Biomedical Sciences, University of Florence, Florence, Italy, ${ }^{6}$ Department of Internal Medicine, Máxima Medical Center, Eindhoven, The Netherlands, ${ }^{7}$ Division of General Internal Medicine, Department of Internal Medicine, Maastricht University Medical Centre+, Maastricht, The Netherlands, ${ }^{8}$ Maastricht University, CAPHRI School for Public Health and Primary Care, Ageing and Long-Term Care, Maastricht, The Netherlands, ${ }^{9}$ Department of Endocrinology and Metabolism, Academic Medical Center, Amsterdam, The Netherlands, ${ }^{10}$ Endocrinology Section, Department of Internal Medicine, VU University Medical Center, Amsterdam, The Netherlands, ${ }^{11}$ Department of Endocrinology, Leiden University Medical Center, Leiden, The Netherlands,

${ }^{12}$ Department of Radiology, Erasmus Medical Center, Rotterdam, The Netherlands, ${ }^{13}$ Department of Radiology, Máxima Medical Center, Eindhoven, The Netherlands, ${ }^{14}$ Department of Radiology, VU University Medical Center, Amsterdam, The Netherlands, and ${ }^{15}$ Department of Radiology and Nuclear Medicine, Academic Medical Center, University of Amsterdam, Amsterdam, The Netherlands
\end{abstract}

\begin{abstract}
Background: A substantial proportion of all pheochromocytomas is currently detected during the evaluation of an adrenal incidentaloma. Recently, it has been suggested that biochemical testing to rule out pheochromocytoma is unnecessary in case of an adrenal incidentaloma with an unenhanced attenuation value $\leq 10$ Hounsfield Units (HU) at computed tomography (CT).

Objectives: We aimed to determine the sensitivity of the $10 \mathrm{HU}$ threshold value to exclude a pheochromocytoma. Methods: Retrospective multicenter study with systematic reassessment of preoperative unenhanced CT scans performed in patients in whom a histopathologically proven pheochromocytoma had been diagnosed. Unenhanced attenuation values were determined independently by two experienced radiologists. Sensitivity of the $10 \mathrm{HU}$ threshold was calculated, and interobserver consistency was assessed using the intraclass correlation coefficient (ICC). Results: 214 patients were identified harboring a total number of 222 pheochromocytomas. Maximum tumor diameter was 51 (39-74) $\mathrm{mm}$. The mean attenuation value within the region of interest was $36 \pm 10 \mathrm{HU}$. Only one pheochromocytoma demonstrated an attenuation value $\leq 10 \mathrm{HU}$, resulting in a sensitivity of $99.6 \%(95 \% \mathrm{Cl}$ : 97.5-99.9). ICC was 0.81 (95\% Cl: 0.75-0.86) with a standard error of measurement of $7.3 \mathrm{HU}$ between observers. Conclusion: The likelihood of a pheochromocytoma with an unenhanced attenuation value $\leq 10 \mathrm{HU}$ on CT is very low. The interobserver consistency in attenuation measurement is excellent. Our study supports the recommendation that in patients with an adrenal incidentaloma biochemical testing for ruling out pheochromocytoma is only indicated in adrenal tumors with an unenhanced attenuation value $>10 \mathrm{HU}$.
\end{abstract}

(C) 2018 European Society of Endocrinology Printed in Great Britain
European Journal of Endocrinology

(2018) 178, 431-437 


\section{Introduction}

Pheochromocytomas are rare neuroendocrine tumors arising from chromaffin tissue in the adrenal medulla (1). Timely diagnosis is crucial because patients are at increased risk for serious cardiovascular complications due to uncontrolled catecholamine hypersecretion $(2,3)$. In general, the presence of a pheochromocytoma is suspected in case of typical signs and symptoms such as paroxysmal headaches, palpitations, sweating and hypertension (4). The diagnosis of a pheochromocytoma is biochemically established by demonstration of elevated plasma or urinary levels of metanephrines, the $O$-methylated metabolites of catecholamines. However, a substantial proportion of patients with pheochromocytoma is either asymptomatic or may present with less typical signs and symptoms $(5,6)$. In such cases, a pheochromocytoma may be detected following the hormonal evaluation of an adrenal incidentaloma, which is defined as an adrenal mass $>1 \mathrm{~cm}$ discovered incidentally during imaging procedures performed for reasons unrelated to adrenal disease (7). The reported prevalence of pheochromocytoma in adrenal incidentaloma cohorts is approximately $5-7 \%(8,9)$. Conversely, nearly $30 \%$ of all pheochromocytomas are currently being diagnosed through the evaluation of adrenal incidentalomas (10). In view of these prevalence data, and the premise that a diagnosis of pheochromocytoma should not be missed, determination of metanephrines is recommended by the majority of guidelines on the management of adrenal incidentalomas $(7,9)$.

It has been suggested, however, that the likelihood of a pheochromocytoma is extremely low when the adrenal tumor demonstrates an unenhanced CT attenuation value $\leq 10$ Hounsfield Units (HU). If this assumption would indeed be true, then expensive measurement of metanephrines could be reserved for adrenal incidentalomas with an unenhanced attenuation value above this threshold $(11,12,13)$. In agreement with these observations, the recent guideline issued by the European Society of Endocrinology/European Network for the Study of Adrenal Tumors (ESE/ENSAT) states that it seems reasonable to avoid measuring metanephrines in case of an adrenal incidentaloma with an unenhanced attenuation value $\leq 10 \mathrm{HU}$ (9). The quality of evidence behind this recommendation is very low, as only a few small-sized studies are available on this subject. Moreover, there are several reports describing pheochromocytomas with an unenhanced attenuation value $\leq 10 \mathrm{HU}$, suggesting that the sensitivity of this cut-off point might be suboptimal for application in clinical practice $(14,15)$.

We therefore aimed to systematically evaluate the characteristics of pheochromocytoma at unenhanced CT scan in a large-sized study in order to determine the diagnostic value of this cut-off value to exclude the presence of a pheochromocytoma.

\section{Subjects and methods}

Patients with histologically proven pheochromocytoma diagnosed between the year 2000 and 2017 were identified in six university medical centers and one nonuniversity teaching hospital in The Netherlands. Patients were considered eligible if the original preoperative unenhanced CT scan images of the adrenal glands were available for analysis. Recurrent or residual tumors were excluded. This retrospective study has been exempted from review and approval by the medical research and ethics committee of the University of Groningen, The Netherlands, according to the Dutch Medical Research Involving Human Subjects Act.

Clinical and biochemical data were extracted from the medical records and were anonymized before further analysis. Selected CT scans were reviewed independently by two experienced radiologists ( $\mathrm{T} \mathrm{K}$ and $\mathrm{C} \mathrm{H}$ ) using a standardized protocol. The radiologists were not blinded for the diagnosis of pheochromocytoma. Scanning acquisition and reconstruction parameters were extracted and the image quality was graded sufficient or insufficient for further analysis. The presence of movement or beam artifacts that interfered with attenuation measurement, incomplete visualization of the tumor and slice thickness $>5 \mathrm{~mm}$ were considered as factors rendering the image quality insufficient. A two-dimensional region of interest (ROI) was drawn in the transversal plane by each radiologist avoiding the edges of the tumor and areas containing necrosis, calcifications and cystic formations. The radiologists had also access to the post-contrast series in case these were performed, which enabled more precise drawing of the ROI. The mean attenuation value, standard deviation of the attenuation value and ROI size were determined. In order to limit interobserver variability, a volume of interest (VOI) encapsulating the whole tumor was generated using a workstation (Syngo.via (version VB10B_HF06) Siemens Healthineers, Erlangen, Germany), which automatically determined the borders of the tumor. In a second step, these borders were visually checked and, 
if needed, manually corrected. The software subsequently calculated the volume in which the mean attenuation value and the standard deviation of the attenuation value were determined. This enabled precise determination of the tumor volume even in case of an irregular shaped adrenal lesion. The standard deviation of each volumetric attenuation value was used as a measure of heterogeneity of the tumor. The amount of necrosis was estimated, and the presence of fine or coarse calcifications was graded.

The primary objective of this study was to determine the sensitivity of the $10 \mathrm{HU}$ cut-off value to demonstrate or exclude the presence of a pheochromocytoma. Sensitivity was calculated as a percentage with 95\% confidence intervals. As secondary objective of this study, we determined concordance between radiologists with respect to classification of the pheochromocytoma (i.e. attenuation $\leq$ or $>10 \mathrm{HU}$ ) using Cohen's Kappa coefficient. Consistency of attenuation measurements between radiologists was assessed using the intraclass correlation coefficient (ICC) and a standard error of measurement. Consistency between the volumetric measurement and transversal measurement was assessed with ICC. Another, secondary objective of this study was to determine potential relationships between the attenuation value, presence of calcifications, tumor necrosis tumor heterogeneity and acquisition and reconstruction parameters using Pearson's or Spearman correlations coefficients were appropriate. Kruskal-Wallis and MannWhitney tests were used to assess the relationship between attenuation value and categorical data where appropriate. The difference between volumetric and transversal attenuation measurement was calculated as a marker of selection bias in the ROI placement. Plasma and urinary metanephrines were calculated as ratios of the upper reference limit maintained by each laboratory and the biochemical phenotype was determined as previously described (16).

Data are represented as mean \pm standard deviation (s.D.) or median (interquartile range) where appropriate. Statistical analyses were performed using SPSS, version 23.0 for Windows (IBM Corporation). A two-sided $P$ value $<0.05$ was considered significant.

\section{Results}

We initially identified 224 eligible patients with pheochromocytoma from whom a preoperative unenhanced CT scan was available. After review, 10 cases were excluded since the pheochromocytoma was not fully

Table 1 Characteristics of 214 study subjects with a pheochromocytoma. Data are represented as mean \pm S.D., median (IQR) or percentages.

Age (years)

$\operatorname{Sex}(\mathrm{m} / \mathrm{f})$

BMI $\left(\mathrm{kg} / \mathrm{m}^{2}\right)$

PCC localization (left/right/bilateral)

Plasma MN (ratio of URL)

Plasma NMN (ratio of URL)

Urinary MN (ratio of URL)

Urinary NMN (ratio of URL)

Biochemical phenotype (A/NA)

$53 \pm 15$
$40 \% / 60 \%$
$54.4(21.8-27.9)$
$47 \% / 49 \% / 4 \%$
$3.9(1.1-14.3)$
$7.9(2.3-18.8)$
$4.1(1.0-14.5)$
$3.6(1.7-7.7)$
$66 \% / 34 \%$

$\mathrm{A}$, adrenergic; BMI, body mass index; $\mathrm{MN}$, metanephrine; NA, noradrenergic; NMN, normetanephrine; PCC, pheochromocytoma; URL, upper reference limit.

imaged in nine cases and the image quality was insufficient in one case. Therefore, 214 patients harboring a total of 222 histologically confirmed pheochromocytomas were included into the final analysis. The mean age of patients was $53 \pm 15$ years, $60 \%$ were female and the biochemical phenotype was predominantly adrenergic (Table 1). In $32 \%$ of cases, the pheochromocytoma was diagnosed during the evaluation of an adrenal incidentaloma. Acquisition and reconstruction characteristics are shown in Table 2. In $89 \%$ of CT scans, tube potential was set at 120 kilovolt $(\mathrm{kV})$ and in $85 \%$ of cases additional postcontrast series were available.

The maximum tumor diameter in any plane was 51 (39-74) $\mathrm{mm}$ and median tumor volume was 31 (13-92) $\mathrm{cm}^{3}$. Mean unenhanced attenuation value within the ROI placed by each radiologist was $36 \pm 9 \mathrm{HU}$ and $37 \pm 9 \mathrm{HU}$ for observer 1 and observer 2 , respectively

Table 2 Acquisition and reconstruction parameters of 214 CT scans. Data are represented as percentage or median (IQR).

\begin{tabular}{lc}
\hline Parameters & Values \\
\hline Slices & \\
16 & $17 \%$ \\
64 & $21 \%$ \\
320 & $14 \%$ \\
Other & $28 \%$ \\
$\quad$ Unknown & $20 \%$ \\
Slice thickness (mm) & $3.0(2.0-3.2)$ \\
Slice increment (mm) & $2.0(1.5-3.0)$ \\
Tube potential (kV) & \\
$\quad<120$ & $7 \%$ \\
120 & $89 \%$ \\
$>120$ & $4 \%$ \\
Reconstruction kernel & \\
Soft & $39 \%$ \\
Medium & $56 \%$ \\
Unknown & $5 \%$ \\
\hline kV, kilovolt. & \\
\hline
\end{tabular}


Table 3 Tumor characteristics and attenuation values of 222 pheochromocytomas. Data are represented as mean \pm S.D., median (IQR) or percentages.

\begin{tabular}{l} 
Tumor characteristics \\
\hline Diameter transversal plane $(\mathrm{mm})$ \\
Orthogonal diameter $(\mathrm{mm})$ \\
Maximum diameter $(\mathrm{mm})$ \\
Calcifications $(\%)$ \\
$\quad$ None \\
Fine \\
Course \\
Necrosis $(\%)$ \\
0 \\
$\leq 10$ \\
$11-50$ \\
$>50$ \\
Region of interest $\left(\mathrm{cm}^{2}\right) *$ \\
Mean attenuation value $(\mathrm{HU})$ \\
S.D. attenuation value $(\mathrm{HU})$
\end{tabular}

\begin{tabular}{c} 
Values \\
\hline $43(33-64)$ \\
$36(25-50)$ \\
$51(39-74)$ \\
93 \\
3 \\
4 \\
51 \\
10 \\
21 \\
18
\end{tabular}

Observer 1

Observer 2

Volumetric

*The region of interest for the volumetric measurement is expressed as $\mathrm{cm}^{3}$. HU, Hounsfield Units.

(Table 3). The ICC was 0.81 (95\% CI: $0.75-0.86)$ and standard error of measurement was $7.3 \mathrm{HU}$ between observers. The unenhanced attenuation value of 221 pheochromocytomas was classified $>10 \mathrm{HU}$ by both radiologists, whereas one pheochromocytoma was judged concordantly $\leq 10 \mathrm{HU}$. This resulted in perfect interobserver reliability: $k=1.00, P<0.001$. The sensitivity of the $10 \mathrm{HU}$ cut-off for identifying a pheochromocytoma was 99.6\% (95\% CI: 97.5-99.9; Figs 1 and 2).

Mean attenuation value in the VOI was $32 \pm 9 \mathrm{HU}$. ICC was 0.75 (95\% CI: $0.24-0.89$ ) and standard error of measurement was $8.4 \mathrm{HU}$ between the volumetric attenuation value and both measurements in the transversal plane. The difference between volumetric and transversal attenuation measurements was positively associated with tumor heterogeneity (standard deviation of the VOI attenuation value, $R_{\mathrm{s}} 0.23, P=0.001$ ) as well as, with the presence of necrosis $\left(R_{\mathrm{s}} 0.32, P<0.001\right)$, but not with tumor volume $\left(R_{\mathrm{s}} 0.06, P=0.399\right)$.

Interrater reliability between radiologists for scoring calcifications and necrosis was $k=0.56$ and $k=0.54$, respectively $(P<0.001$ for both). The mean attenuation value in the transversal plane was not significantly associated with tumor volume, presence of calcifications, tube potential or tumor heterogeneity (data not shown). The mean attenuation value in the transversal plane was lower in tumors containing more than 50\% necrosis (mean attenuation $37 \pm 9,38 \pm 6,38 \pm 7$ and $33 \pm 7 \mathrm{HU}$ for $0,1-10,11-50$ and $>50 \%$ necrosis respectively, $P=0.029$ ).

The single patient with an unenhanced attenuation value $\leq 10 \mathrm{HU}$ was found to have Cushing's syndrome caused by ectopic ACTH secretion from a left-sided pheochromocytoma. The diagnosis of pheochromocytoma was not considered before surgery, but was established by pathological examination of the resected adrenal gland, demonstrating a $3.5 \mathrm{~cm}$ large tumor consisting of cell nests with eosinophilic cytoplasm, with positive immunohistochemical staining for chromogranine, synaptophysine, S-100 and ACTH. Preoperative

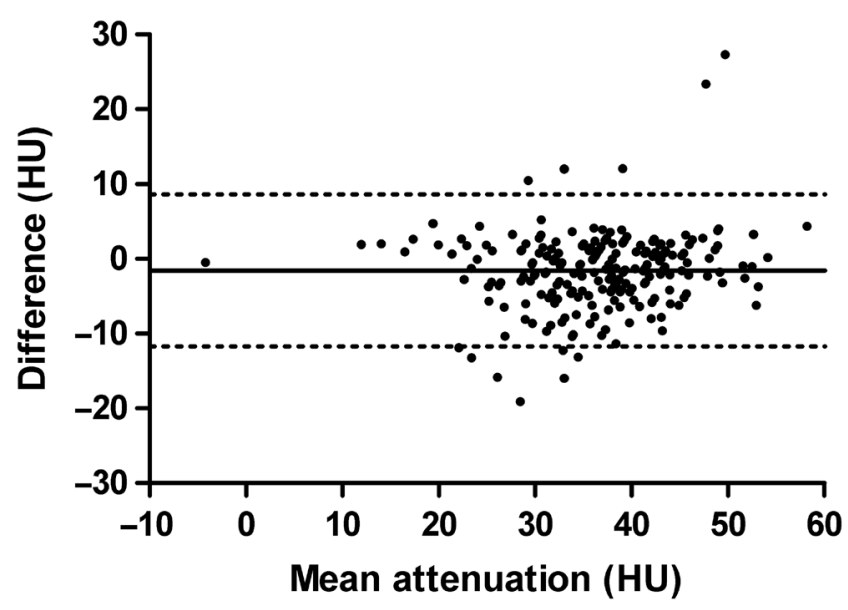

Figure 1

Bland-Altman plot of 222 pheochromocytoma showing the mean value of attenuation measurements by two radiologists from a single patient (X-axis) plotted against the difference between the same two results (Y-axis). The solid horizontal line represents the mean difference of all measurements with corresponding $95 \%$ limits of agreement represented as the dotted lines. 


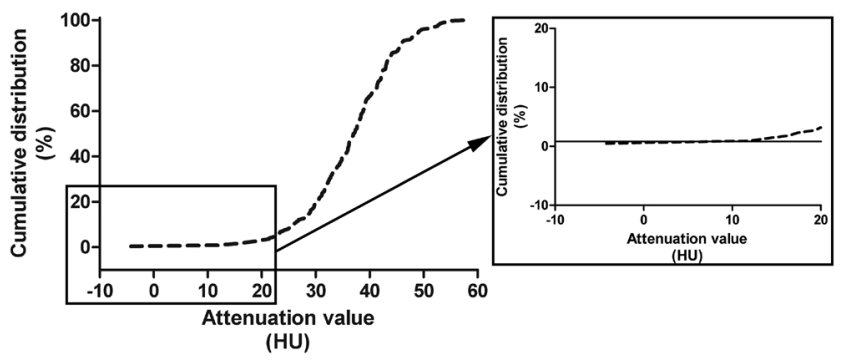

Figure 2

Cumulative distribution in percentage ( $Y$-axis) displayed for the mean attenuation value (X-axis) for 222

pheochromocytomas on the left. On the right side, a magnification of the lower range of attenuation values including the intersection with the $10 \mathrm{HU}$ cut-off value.

urinary free cortisol excretion was $13000 \mathrm{nmol} / 24 \mathrm{~h}$. The unenhanced attenuation value measured by both radiologists was $-4 \mathrm{HU}$ and the VOI attenuation value was $-15 \mathrm{HU}$. Remarkably, the standard deviation of the attenuation value of this tumor represented the highest value of the entire study population (i.e. $66 \mathrm{HU}$ ), despite the absence of necrosis, cystic parts or calcifications (Fig. 3).

\section{Discussion}

In this large retrospective multicenter study, we show that the finding of an unenhanced attenuation value $\leq 10 \mathrm{HU}$ in an adrenal gland tumor has a high diagnostic value of

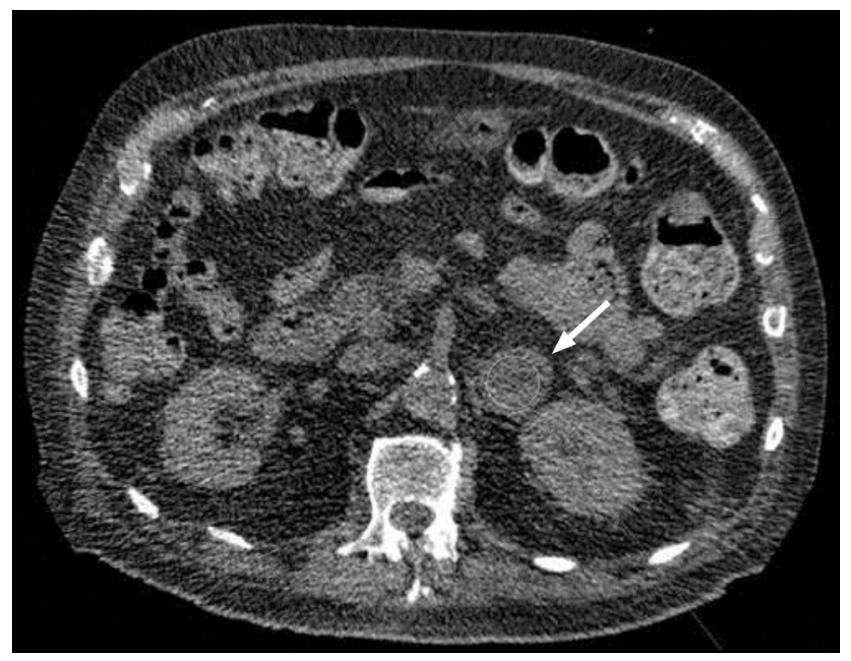

\section{Figure 3}

Left-sided pheochromocytoma with ectopic ACTH production demonstrating an unenhanced attenuation value of $-4 \mathrm{HU}$.
99.6\% to exclude the presence of a pheochromocytoma. Moreover, the applicability of this method is underlined by the excellent interobserver agreement.

Over the past two decades, there has been a rapid increase in the utilization of various imaging techniques, which has led to a marked rise in the detection rate of adrenal incidentalomas. Radiological studies report a frequency varying from $3 \%$ to $10 \%$, with the highest rates among the elderly $(9,17,18,19)$. The detection of an adrenal incidentaloma should be followed by further analysis in order to evaluate whether the lesion is hormonal active or not and whether the adrenal tumor is benign or malignant. For this purpose, several algorithms have been developed $(7,9,20)$. One of the common denominators in all these algorithms is the recommendation to measure metanephrines in plasma or urine in order to exclude the presence of a pheochromocytoma. However, the recently issued ESE/ENSAT guideline has suggested that determination of metanephrines might be obviated in case of an adrenal incidentaloma with an unenhanced CT attenuation value $\leq 10 \mathrm{HU}$ (9). This recommendation was based on small-sized studies predominantly describing pheochromocytomas with an unenhanced attenuation value $>10 \mathrm{HU}(21,22)$. In agreement with these earlier reports, we demonstrate that pheochromocytomas with low unenhanced attenuation values are a very infrequent phenomenon.

Biochemical tests for pheochromocytoma have an excellent negative predictive value of 0.99 (23). However, the specificity depends quite strongly on preanalytical conditions, such as the use of certain drugs (e.g. tricyclic antidepressants, dopamine D2 receptor antagonists) and the requirement of a correctly collected 24-h urine or blood sampling after at least $20 \mathrm{~min}$ of supine rest for measurement of metanephrines in urine or plasma, respectively (24). In addition, determination of metanephrines is rather expensive. As it is not always feasible to create optimal preanalytical conditions the rate of false-positive measurements might be increased, subsequently resulting in more diagnostic testing to rule out pheochromocytoma at higher costs (25). Moreover, almost $70 \%$ of adrenal incidentalomas demonstrate attenuation values $\leq 10 \mathrm{HU}$, illustrating the large proportion of patients that might benefit from using the attenuation threshold in order to determine which patients need biochemical screening as second-line testing to rule out pheochromocytoma (26). Notably, approximately 3500 patients with adrenal incidentaloma and attenuation value $\leq 10 \mathrm{HU}$ would need to be biochemically screened in order to diagnose one case of pheochromocytoma, 
assuming a 5\% prevalence of pheochromocytoma in the adrenal incidentaloma population, 70\% frequency of attenuation $\leq 10 \mathrm{HU}$ and a false-negative rate of $0.4 \%$ of radiological classification as determined in the present study. We cannot exclude, however, the possibility that under certain clinical circumstances determination of metanephrines might still be indicated in case of an adrenal mass with a attenuation $<10 \mathrm{HU}$, e.g. in a patient with symptoms suggestive of a pheochromocytoma or when biopsy or adrenalectomy is considered.

The single patient with a false-negative CT scan had an unusual clinical picture of Cushing's syndrome due to ectopic ACTH production by a pheochromocytoma. In this case, the mean attenuation value might have been decreased as a result of the higher cholesterol content associated with the profound hypersecretion of cortisol as is also supported by the extremely high standard deviation of the attenuation value.

Our data further illustrate that clinical applicability of this measurement in daily practice is very reliable if basic rules of ROI drawing are taken into account (27). This is underlined by good interobserver consistency and excellent concordance between radiologists. Selective ROI placement was shown to be especially valuable in heterogeneous tumors with more necrosis. It has been shown that acquisition and reconstruction parameters of CT scans, such as tube potential and scanner type, can induce small changes in attenuation values and a lack of uniformity in these settings might be considered a shortcoming of the current study (28). For example, a significant proportion of adrenal incidentalomas was reclassified over time when using the $10 \mathrm{HU}$ cut-off value due to variability between CT scanners and observer (29). However, the magnitude of this variability is very low and our data show that only a small proportion of pheochromocytomas demonstrates an attenuation value close to $10 \mathrm{HU}$. This suggests that differences in acquisition and reconstruction parameters or interobserver variability are unlikely to affect the radiologic classification of these adrenal tumors (28).

The major strengths of the current study are the systematic reassessment by two independent radiologists performing attenuation measurements in accordance with daily practice in a relatively large number of pheochromocytomas. A limitation of our study is the possibility of confirmation bias due to the non-blinded design or incorporation bias as CT is part of routine diagnostic work-up in case of a pheochromocytoma. Also, its retrospective design, and the fact that we were not able to determine the specificity of the HU threshold value since no control group was included might be considered as a limitation. It should be noted, however, that the specificity of the $10 \mathrm{HU}$ cut-off value is known to be very low for diagnosing a pheochromocytoma (30).

In conclusion, the likelihood that an adrenal incidentaloma with an unenhanced CT attenuation value $\leq 10 \mathrm{HU}$ represents a pheochromocytoma is very low. Our results do not support the widespread clinical practice to determine metanephrines in every patient with an adrenal incidentaloma.

\section{Declaration of interest}

The authors declare that there is no conflict of interest that could be perceived as prejudicing the impartiality of this study.

\section{Funding}

This research did not receive any specific grant from any funding agency in the public, commercial or not-for-profit sector.

\section{References}

1 DeLellis R, Heitz P \& Eng C. Pathology and Genetics: Tumours of Endocrine Organs (IARC WHO Classification of Tumours). Lyon, France: IARC Press, 2004

2 Stolk RF, Bakx C, Mulder J, Timmers HJ \& Lenders JW. Is the excess cardiovascular morbidity in pheochromocytoma related to blood pressure or to catecholamines? Journal of Clinical Endocrinology and Metabolism 201398 1100-1106. (https://doi.org/10.1210/jc.20123669)

3 Zelinka T, Petrak O, Turkova H, Holaj R, Strauch B, Krsek M, Vrankova AB, Musil Z, Duskova J, Kubinyi J et al. High incidence of cardiovascular complications in pheochromocytoma. Hormone and Metabolic Research 201244 379-384. (https://doi. org/10.1055/s-0032-1306294)

4 Lenders JW, Eisenhofer G, Mannelli M \& Pacak K. Phaeochromocytoma. Lancet 2005366 665-675. (https://doi. org/10.1016/S0140-6736(05)67139-5)

5 Mannelli M, Lenders JW, Pacak K, Parenti G \& Eisenhofer G. Subclinical phaeochromocytoma. Best Practice and Research Clinical Endocrinology and Metabolism 201226 507-515.

6 Mannelli M, Ianni L, Cilotti A \& Conti A. Pheochromocytoma in Italy: a multicentric retrospective study. European Journal of Endocrinology 1999141 619-624. (https://doi.org/10.1530/ eje.0.1410619)

7 Young WF Jr. Clinical practice. The incidentally discovered adrenal mass. New England Journal of Medicine 2007356 601-610. (https:// doi.org/10.1056/NEJMcp065470)

8 Barzon L, Sonino N, Fallo F, Palu G \& Boscaro M. Prevalence and natural history of adrenal incidentalomas. European Journal of Endocrinology 2003149 273-285. (https://doi.org/10.1530/ eje.0.1490273)

9 Fassnacht M, Arlt W, Bancos I, Dralle H, Newell-Price J, Sahdev A, Tabarin A, Terzolo M, Tsagarakis S \& Dekkers OM. Management of adrenal incidentalomas: European Society of Endocrinology Clinical Practice Guideline in collaboration with the European Network for the Study of Adrenal Tumors. European Journal of Endocrinology 2016 175 G1-G34. (https://doi.org/10.1530/EJE-16-0467)

10 Kopetschke R, Slisko M, Kilisli A, Tuschy U, Wallaschofski H, Fassnacht M, Ventz M, Beuschlein F, Reincke M, Reisch N \& 
Quinkler M. Frequent incidental discovery of phaeochromocytoma: data from a German cohort of 201 phaeochromocytoma. European Journal of Endocrinology 2009161 355-361. (https://doi.org/10.1530/ EJE-09-0384)

11 Sane T, Schalin-Jantti C \& Raade M. Is biochemical screening for pheochromocytoma in adrenal incidentalomas expressing low unenhanced attenuation on computed tomography necessary? Journal of Clinical Endocrinology and Metabolism 201297 2077-2083. (https://doi.org/10.1210/jc.2012-1061)

12 Schalin-Jantti C, Raade M, Hamalainen E \& Sane T. A 5-year prospective follow-up study of lipid-rich adrenal incidentalomas: no tumor growth or development of hormonal hypersecretion. Endocrinology and Metabolism 201530 481-487. (https://doi. org/10.3803/EnM.2015.30.4.481)

13 Patel J, Davenport MS, Cohan RH \& Caoili EM. Can established CT attenuation and washout criteria for adrenal adenoma accurately exclude pheochromocytoma? American Journal of Roentgenology 2013 201 122-127. (https://doi.org/10.2214/AJR.12.9620)

14 Blake MA, Krishnamoorthy SK, Boland GW, Sweeney AT, Pitman MB, Harisinghani M, Mueller PR \& Hahn PF. Low-density pheochromocytoma on CT: a mimicker of adrenal adenoma. American Journal of Roentgenology 2003181 1663-1668.

15 Jun JH, Ahn HJ, Lee SM, Kim JA, Park BK, Kim JS \& Kim JH. Is preoperative biochemical testing for pheochromocytoma necessary for all adrenal incidentalomas? Medicine 201594 e1948.

16 Gupta G, Pacak K \& AACE Adrenal Scientific Committee. Precision medicine: an update on genotype/biochemical phenotype relationships in pheochromocytoma/paraganglioma patients. Endocrine Practice 2017 23 690-704. (https://doi.org/10.4158/EP161718.RA)

17 Zeiger MA, Siegelman SS \& Hamrahian AH. Medical and surgical evaluation and treatment of adrenal incidentalomas. Journal of Clinical Endocrinology and Metabolism 201196 2004-2015.

18 Smith-Bindman R, Miglioretti DL, Johnson E, Lee C, Feigelson HS, Flynn M, Greenlee RT, Kruger RL, Hornbrook MC, Roblin D et al. Use of diagnostic imaging studies and associated radiation exposure for patients enrolled in large integrated health care systems, 1996-2010. JAMA 2012307 2400-2409.

19 Bijwaard H, Pruppers M \& de Waard-Schalkx I. The influence of population aging and size on the number of CT examinations in The Netherlands. Health Physics 2014107 80-82. (https://doi. org/10.1097/HP.0000000000000050)

20 Terzolo M, Stigliano A, Chiodini I, Loli P, Furlani L, Arnaldi G, Reimondo G, Pia A, Toscano V, Zini M et al. AME position statement on adrenal incidentaloma. European Journal of Endocrinology 2011164 851-870. (https://doi.org/10.1530/EJE-10-1147)
21 Kannan S, Purysko A, Faiman C, Remer EM, Shah L, Bena J, Siperstein A, Berber E, Fergany A, Bravo E et al. Biochemical and radiological relationships in patients with pheochromocytoma: lessons from a case control study. Clinical Endocrinology 201480 790-796. (https://doi.org/10.1111/cen.12420)

22 Motta-Ramirez GA, Remer EM, Herts BR, Gill IS \& Hamrahian AH. Comparison of CT findings in symptomatic and incidentally discovered pheochromocytomas. American Journal of Roentgenology 2005185 684-688. (https://doi.org/10.2214/ ajr.185.3.01850684)

23 Darr R, Kuhn M, Bode C, Bornstein SR, Pacak K, Lenders JWM \& Eisenhofer G. Accuracy of recommended sampling and assay methods for the determination of plasma-free and urinary fractionated metanephrines in the diagnosis of pheochromocytoma and paraganglioma: a systematic review. Endocrine 201756 495-503. (https://doi.org/10.1007/s12020-017-1300-y)

24 Lenders JW, Duh QY, Eisenhofer G, Gimenez-Roqueplo AP, Grebe SK, Murad MH, Naruse M, Pacak K, Young WF Jr \& Endocrine Society. Pheochromocytoma and paraganglioma: an endocrine society clinical practice guideline. Journal of Clinical Endocrinology and Metabolism 201499 1915-1942.

25 Lenders JW, Willemsen JJ, Eisenhofer G, Ross HA, Pacak K, Timmers HJ \& Sweep CG. Is supine rest necessary before blood sampling for plasma metanephrines? Clinical Chemistry $2007 \mathbf{5 3}$ 352-354. (https://doi.org/10.1373/clinchem.2006.076489)

26 Bancos I, Chortis V, Lang K, Prete A, Terzolo M, Fassnacht M, Quinkler M, Kastelan D, Vassiliadi D, Beuschlein F et al. The natural history of adrenal incidentaloma - results from the international prospective multi-centre EURINE-ACT study. Endocrine Abstracts 2017 49 GP122. (https://doi.org/10.1530/endoabs.49.GP122)

27 Blake MA, Cronin CG \& Boland GW. Adrenal imaging. American Journal of Roentgenology 2010194 1450-1460. (https://doi. org/10.2214/AJR.10.4547)

28 Cropp RJ, Seslija P, Tso D \& Thakur Y. Scanner and kVp dependence of measured CT numbers in the ACR CT phantom. Journal of Applied Clinical Medical Physics 201314 4417. (https://doi.org/10.1120/ jacmp.v14i6.4417)

29 Hammarstedt L, Thilander-Klang A, Muth A, Wangberg B, Oden A \& Hellstrom M. Adrenal lesions: variability in attenuation over time, between scanners, and between observers. Acta Radiologica 201354 817-826. (https://doi.org/10.1177/0284185113482688)

30 Jun JH, Ahn HJ, Lee SM, Kim JA, Park BK, Kim JS \& Kim JH. Is preoperative biochemical testing for pheochromocytoma necessary for all adrenal incidentalomas? Medicine 201594 e1948. (https://doi. org/10.1097/MD.0000000000001948)

Received 5 January 2018

Revised version received 14 February 2018

Accepted 20 February 2018 